PROCEEDINGS OF THE

AMERICAN MATHEMATICAL SOCIETY

Volume 137, Number 3, March 2009, Pages 1025-1034

S 0002-9939(08)09717-7

Article electronically published on October 17, 2008

\title{
DYNAMICS OF TUPLES OF MATRICES
}

\author{
G. COSTAKIS, D. HADJILOUCAS, AND A. MANOUSSOS
}

(Communicated by Nigel J. Kalton)

\begin{abstract}
In this article we answer a question raised by N. Feldman in 2008 concerning the dynamics of tuples of operators on $\mathbb{R}^{n}$. In particular, we prove that for every positive integer $n \geq 2$ there exist $n$-tuples $\left(A_{1}, A_{2}, \ldots, A_{n}\right)$ of $n \times n$ matrices over $\mathbb{R}$ such that $\left(A_{1}, A_{2}, \ldots, A_{n}\right)$ is hypercyclic. We also establish related results for tuples of $2 \times 2$ matrices over $\mathbb{R}$ or $\mathbb{C}$ being in Jordan form.
\end{abstract}

\section{INTRODUCTION}

Following the recent work of Feldman in [4 an $n$-tuple of operators is a finite sequence of length $n$ of commuting continuous linear operators $T_{1}, T_{2}, \ldots, T_{n}$ acting on a locally convex space $X$. The tuple $\left(T_{1}, T_{2}, \ldots, T_{n}\right)$ is hypercyclic if there exists a vector $x \in X$ such that the set

$$
\left\{T_{1}^{k_{1}} T_{2}^{k_{2}} \cdots T_{n}^{k_{n}} x: k_{1}, k_{2}, \ldots, k_{n} \geq 0\right\}
$$

is dense in $X$. Such a vector $x$ is called hypercyclic for $\left(T_{1}, T_{2}, \ldots, T_{n}\right)$ and the set of hypercyclic vectors for $\left(T_{1}, T_{2}, \ldots, T_{n}\right)$ will be denoted by $H C\left(\left(T_{1}, T_{2}, \ldots, T_{n}\right)\right)$. The above definition generalizes the notion of hypercyclicity to tuples of operators. For an account of results, comments and an extensive bibliography on hypercyclicity we refer to [1, [5, 6] and [7. For results concerning the dynamics of tuples of operators see [2, [3, 4] and 9].

In 4] Feldman showed, among other things, that in $\mathbb{C}^{n}$ there exist diagonalizable $(n+1)$-tuples of matrices having dense orbits. In addition he proved that there is no $n$-tuple of diagonalizable matrices on $\mathbb{R}^{n}$ or $\mathbb{C}^{n}$ that has a somewhere dense orbit. Therefore the following question arose naturally.

Question (Feldman 4]). Are there non-diagonalizable $n$-tuples on $\mathbb{R}^{k}$ that have somewhere dense orbits?

We give a positive answer to this question in a very strong form, as the next theorem shows.

Received by the editors March 24, 2008.

2000 Mathematics Subject Classification. Primary 47A16.

Key words and phrases. Hypercyclic operators, tuples of matrices.

During this research the third author was fully supported by SFB 701 "Spektrale Strukturen und Topologische Methoden in der Mathematik" at the University of Bielefeld, Germany. He would also like to express his gratitude to Professor H. Abels for his support.

(C)2008 American Mathematical Society 
Theorem 1.1. For every positive integer $n \geq 2$ there exist $n$-tuples $\left(A_{1}, \ldots, A_{n}\right)$ of $n \times n$ non-(simultaneously) diagonalizable matrices over $\mathbb{R}$ such that $\left(A_{1}, \ldots, A_{n}\right)$ is hypercyclic.

Restricting ourselves to tuples of $2 \times 2$ matrices in Jordan form either on $\mathbb{R}^{2}$ or $\mathbb{C}^{2}$, we prove the following.

Theorem 1.2. There exist $2 \times 2$ matrices $A_{j}, j=1,2,3,4$, in Jordan form over $\mathbb{R}$ such that $\left(A_{1}, A_{2}, A_{3}, A_{4}\right)$ is hypercyclic. In particular

$$
H C\left(\left(A_{1}, A_{2}, A_{3}, A_{4}\right)\right)=\left\{\left(\begin{array}{c}
x_{1} \\
x_{2}
\end{array}\right) \in \mathbb{R}^{2}: x_{2} \neq 0\right\} .
$$

Theorem 1.3. There exist $2 \times 2$ matrices $A_{j}, j=1,2, \ldots, 8$, in Jordan form over $\mathbb{C}$ such that $\left(A_{1}, A_{2}, \ldots, A_{8}\right)$ is hypercyclic.

\section{Products of $2 \times 2$ Matrices}

Lemma 2.1. Let $m$ be a positive integer and for each $j=1,2, \ldots, m$ let $A_{j}$ be a $2 \times 2$ matrix in Jordan form over a field $\mathbb{F}=\mathbb{C}$ or $\mathbb{R}$, i.e. $A_{j}=\left(\begin{array}{cc}a_{j} & 1 \\ 0 & a_{j}\end{array}\right)$ for $a_{1}, a_{2}, \ldots, a_{m} \in \mathbb{F}$. Then $\left(A_{1}, A_{2}, \ldots, A_{m}\right)$ over $\mathbb{C}$ (respectively $\left.\mathbb{R}\right)$ is hypercyclic if and only if the sequence

$$
\left\{\left(\begin{array}{c}
\frac{k_{1}}{a_{1}}+\frac{k_{2}}{a_{2}}+\ldots+\frac{k_{m}}{a_{m}} \\
a_{1} k_{1} a_{2} k_{2} \ldots a_{m} k_{m}
\end{array}\right): k_{1}, k_{2}, \ldots, k_{m} \in \mathbb{N}\right\}
$$

is dense in $\mathbb{C}^{2}$ (respectively $\mathbb{R}^{2}$ ).

Proof. We prove the above in the case $\mathbb{F}=\mathbb{C}$, since the other case is similar. Observe that

$$
A_{j}^{l}=\left(\begin{array}{cc}
a_{j}^{l} & l a_{j}^{l-1} \\
0 & a_{j}^{l}
\end{array}\right)
$$

for $l \in \mathbb{N}$. As a result we have

$$
A_{1}{ }^{k_{1}} A_{2}{ }^{k_{2}} \ldots A_{m}{ }^{k_{m}}=\left(\begin{array}{cc}
\prod_{j=1}^{m} a_{j}{ }^{k_{j}} & \prod_{j=1}^{m} a_{j}{ }^{k_{j}} \sum_{s=1}^{m} \frac{k_{s}}{a_{s}} \\
0 & \prod_{j=1}^{m} a_{j}^{k_{j}}
\end{array}\right) .
$$

Assume that $\left(A_{1}, A_{2}, \ldots, A_{m}\right)$ is hypercyclic and let $\left(\begin{array}{c}z_{1} \\ z_{2}\end{array}\right) \in \mathbb{C}^{2}$ be a hypercyclic vector for $\left(A_{1}, A_{2}, \ldots, A_{m}\right)$. Then the sequence

$$
\begin{aligned}
& \left\{A_{1}{ }^{k_{1}} A_{2}{ }^{k_{2}} \ldots A_{m}{ }^{k_{m}}\left(\begin{array}{c}
z_{1} \\
z_{2}
\end{array}\right): k_{1}, k_{2}, \ldots, k_{m} \in \mathbb{N}\right\} \\
& =\left\{\left(\begin{array}{c}
z_{1} \prod_{j=1}^{m} a_{j}{ }^{k_{j}}+z_{2} \prod_{j=1}^{m} a_{j}{ }^{k_{j}} \sum_{s=1}^{m} \frac{k_{s}}{a_{s}} \\
z_{2} \prod_{j=1}^{m} a_{j}^{k_{j}}
\end{array}\right): k_{1}, k_{2}, \ldots, k_{m} \in \mathbb{N}\right\}
\end{aligned}
$$

is dense in $\mathbb{C}^{2}$. This implies that $z_{2} \neq 0$. Dividing the element in the first row by that in the second, it can easily be shown that the sequence

$$
\left\{\left(\begin{array}{c}
\frac{k_{1}}{a_{1}}+\frac{k_{2}}{a_{2}}+\ldots+\frac{k_{m}}{a_{m}} \\
a_{1} k_{1} a_{2} k_{2} \ldots a_{m} k_{m}
\end{array}\right): k_{1}, k_{2}, \ldots, k_{m} \in \mathbb{N}\right\}
$$

is dense in $\mathbb{C}^{2}$. The converse can easily be shown. 
Remark 2.2. Let $m$ be a positive integer and for each $j=1,2, \ldots, m$ let $A_{j}$ be a $2 \times 2$ matrix in Jordan form over a field $\mathbb{F}=\mathbb{C}$ or $\mathbb{R}$. By the proof of Lemma2.1 it is immediate that whenever $\left(A_{1}, A_{2}, \ldots, A_{m}\right)$ over $\mathbb{C}$ (respectively $\mathbb{R}$ ) is hypercyclic, one can completely describe the set of hypercyclic vectors as

$$
\left\{\left(\begin{array}{l}
z_{1} \\
z_{2}
\end{array}\right) \in \mathbb{C}^{2}: z_{2} \neq 0\right\} \quad\left(\text { respectively }\left\{\left(\begin{array}{l}
x_{1} \\
x_{2}
\end{array}\right) \in \mathbb{R}^{2}: x_{2} \neq 0\right\}\right) .
$$

2.1. The real case. The one-dimensional version of Kronecker's theorem stated below (see for example [8, Theorem 438, p. 375]) will be used repeatedly throughout this work.

Theorem 2.3. If $x$ is a positive irrational number, then the sequence $\{k x-s$ : $k, s \in \mathbb{N}\}$ is dense in $\mathbb{R}$.

Remark 2.4. If $x$ is a positive irrational number, then the sequence $\{s-k x: k, s \in$ $\mathbb{N}\}$ is also dense in $\mathbb{R}$. Likewise, if $x$ is a negative irrational number, then the sequence $\{s+k x: k, s \in \mathbb{N}\}$ is dense in $\mathbb{R}$.

We shall need the following well-known result; see for example [4].

Theorem 2.5. If $a, b>1$ and $\frac{\ln a}{\ln b}$ is irrational, then the sequence $\left\{\frac{a^{n}}{b^{m}}: n, m \in \mathbb{N}\right\}$ is dense in $\mathbb{R}^{+}$.

Lemma 2.6. Let $a, b \in \mathbb{R}$ such that $-1<a<0, b>1$ and $\frac{\ln |a|}{\ln b}$ is irrational. Then the sequence $\left\{a^{n} b^{m}: n, m \in \mathbb{N}\right\}$ is dense in $\mathbb{R}$.

Proof. Since $\frac{\ln |a|}{\ln b}$ is irrational it follows that $\ln b / \ln \frac{1}{a^{2}}$ is irrational as well. Applying Theorem [2.5 we conclude that the sequence $\left\{a^{2 n} b^{m}: n, m \in \mathbb{N}\right\}$ is dense in $\mathbb{R}^{+}$. On the other hand the fact that $a$ is negative implies that the sequence $\left\{a^{2 n+1} b^{m}: n, m \in \mathbb{N}\right\}$ is dense in $\mathbb{R}^{-}$. This completes the proof of the lemma.

Proposition 2.7. There exist $a_{1}, a_{2}, a_{3}, a_{4} \in \mathbb{R}$ such that the sequence

$$
\left\{\left(\begin{array}{c}
\frac{k_{1}}{a_{1}}+\frac{k_{2}}{a_{2}}+\frac{k_{3}}{a_{3}}+\frac{k_{4}}{a_{4}} \\
a_{1} k_{1} a_{2} k_{2} a_{3}{ }^{k_{3}} a_{4} k_{4}
\end{array}\right): k_{1}, k_{2}, k_{3}, k_{4} \in \mathbb{N}\right\}
$$

is dense in $\mathbb{R}^{2}$.

Proof. By the lemma above fix $a, b \in \mathbb{R}$ such that $-1<a<0, a+\frac{1}{a} \in \mathbb{R} \backslash \mathbb{Q}$ and $\left\{a^{n} b^{m}: n, m \in \mathbb{N}\right\}$ is dense in $\mathbb{R}$. Let $x_{1}, x_{2} \in \mathbb{R}$ and $\epsilon>0$ be given. Then there exist $n, m \in \mathbb{N}$ such that $\left|a^{n} b^{m}-x_{2}\right|<\epsilon$. Note that $a^{n} b^{m}=a^{n+k} b^{m} \frac{1}{a^{k}} 1^{s}$ for every $k, s \in \mathbb{N}$. Note also that $a+\frac{1}{a}<0$. Hence, by Remark 2.4, the sequence

$$
\left\{s+k\left(a+\frac{1}{a}\right): k, s \in \mathbb{N}\right\}
$$

is dense in $\mathbb{R}$; i.e. there exist $k, s \in \mathbb{N}$ such that

$$
\left|s+k\left(a+\frac{1}{a}\right)-\left(x_{1}-\frac{n}{a}-\frac{m}{b}\right)\right|<\epsilon,
$$

i.e.

$$
\left|\frac{n}{a}+\frac{m}{b}+k\left(a+\frac{1}{a}\right)+s-x_{1}\right|<\epsilon .
$$

Hence, setting $a_{1}=a, a_{2}=b, a_{3}=\frac{1}{a}, a_{4}=1$ we prove the result. 
Proof of Theorem 1.2. This is an immediate consequence of Lemma 2.1 Proposition 2.7 and Remark 2.2 ,

Example 2.8. One may construct many concrete examples of four $2 \times 2$ matrices, in Jordan form over $\mathbb{R}$, being hypercyclic. For example, fix $a, b \in \mathbb{R}$ such that $-1<a<0, b>1$ and both $a+\frac{1}{a}, \frac{\ln |a|}{\ln b}$ are irrational. From the above we conclude that

$$
\left(\left(\begin{array}{ll}
a & 1 \\
0 & a
\end{array}\right),\left(\begin{array}{cc}
b & 1 \\
0 & b
\end{array}\right),\left(\begin{array}{cc}
\frac{1}{a} & 1 \\
0 & \frac{1}{a}
\end{array}\right),\left(\begin{array}{cc}
1 & 1 \\
0 & 1
\end{array}\right)\right)
$$

is hypercyclic.

We shall now prove Theorem 1.1 for $n=2$; see Proposition 2.10 (ii). For this we need the following result due to Feldman; see Corollary 3.2 in [4].

Proposition 2.9 (Feldman). Let $\mathbb{D}$ denote the open unit disk centered at 0 in the complex plane. If $b \in \mathbb{D} \backslash\{0\}$, then there exists a dense set $\Delta \subset \mathbb{C} \backslash \mathbb{D}$ such that for every $a \in \Delta$ the sequence $\left\{a^{n} b^{m}: n, m \in \mathbb{N}\right\}$ is dense in $\mathbb{C}$.

Proposition 2.10. (i) Every pair $\left(A_{1}, A_{2}\right)$ of $2 \times 2$ matrices over $\mathbb{R}$ with $A_{j}$, $j=1,2$, being either diagonal or in Jordan form is not hypercyclic.

(ii) There exist pairs $\left(A_{1}, A_{2}\right)$ of $2 \times 2$ matrices over $\mathbb{R}$ such that $A_{1}$ is diagonal, $A_{2}$ is antisymmetric (rotation matrix) and $\left(A_{1}, A_{2}\right)$ is hypercyclic. In particular every non-zero vector in $\mathbb{R}^{2}$ is hypercyclic for $\left(A_{1}, A_{2}\right)$; i.e.

$$
H C\left(\left(A_{1}, A_{2}\right)\right)=\mathbb{R}^{2} \backslash\{(0,0)\} .
$$

(iii) There exist pairs $\left(A_{1}, A_{2}\right)$ of $2 \times 2$ matrices over $\mathbb{R}$ such that both $A_{1}$ and $A_{2}$ are antisymmetric and $\left(A_{1}, A_{2}\right)$ is hypercyclic. In particular every non-zero vector in $\mathbb{R}^{2}$ is hypercyclic for $\left(A_{1}, A_{2}\right)$, i.e.

$$
H C\left(\left(A_{1}, A_{2}\right)\right)=\mathbb{R}^{2} \backslash\{(0,0)\} .
$$

Proof. Let us prove assertion $(i)$. The case of $A_{1}, A_{2}$ both diagonal is covered by Feldman; see 4 .

Assume that $A_{1}$ is diagonal and $A_{2}$ is in Jordan form; i.e.

$$
A_{1}=\left(\begin{array}{cc}
a & 0 \\
0 & a
\end{array}\right), \quad A_{2}=\left(\begin{array}{cc}
b & 1 \\
0 & b
\end{array}\right) \text { for } a, b \in \mathbb{R} .
$$

Suppose that $\left(A_{1}, A_{2}\right)$ is hypercyclic and let $\left(\begin{array}{c}x_{1} \\ x_{2}\end{array}\right) \in \mathbb{R}^{2}$ be a hypercyclic vector for $\left(A_{1}, A_{2}\right)$. Then the sequence

$$
\left\{A_{1}{ }^{n} A_{2}{ }^{m}\left(\begin{array}{l}
x_{1} \\
x_{2}
\end{array}\right): n, m \in \mathbb{N}\right\}=\left\{\left(\begin{array}{c}
a^{n} b^{m} x_{1}+m a^{n} b^{m-1} x_{2} \\
a^{n} b^{m} x_{2}
\end{array}\right): n, m \in \mathbb{N}\right\}
$$

is dense in $\mathbb{R}^{2}$. Therefore $b$ cannot be zero. Observe that $x_{2}$ cannot be zero either. Take any $y_{1} \in \mathbb{R}$ and $y_{2} \in \mathbb{R} \backslash\{0\}$. Then there exist sequences of positive integers $\left\{n_{k}\right\},\left\{m_{k}\right\}$ such that $m_{k} \rightarrow+\infty$ and

$$
\begin{aligned}
& a^{n_{k}} b^{m_{k}} x_{1}+m_{k} a^{n_{k}} b^{m_{k}-1} x_{2} \rightarrow y_{1}, \\
& a^{n_{k}} b^{m_{k}} x_{2} \rightarrow y_{2}
\end{aligned}
$$

as $k \rightarrow+\infty$. Since $b \neq 0, y_{2} \neq 0$ and $x_{2} \neq 0$ we get that

$$
a^{n_{k}} b^{m_{k}} x_{1} \rightarrow \frac{y_{2} x_{1}}{x_{2}} \quad \text { and } \quad\left|m_{k} a^{n_{k}} b^{m_{k}-1} x_{2}\right|=\frac{m_{k}}{|b|}\left|a^{n_{k}} b^{m_{k}} x_{2}\right| \rightarrow+\infty
$$


as $k \rightarrow+\infty$. From the last, it clearly follows that

$$
\left|a^{n_{k}} b^{m_{k}} x_{1}+m_{k} a^{n_{k}} b^{m_{k}-1} x_{2}\right| \rightarrow+\infty,
$$

which is a contradiction.

Assume now that both $A_{1}, A_{2}$ are in Jordan form; i.e.

$$
A_{1}=\left(\begin{array}{cc}
a & 1 \\
0 & a
\end{array}\right), \quad A_{2}=\left(\begin{array}{cc}
b & 1 \\
0 & b
\end{array}\right),
$$

for $a, b \in \mathbb{R}$ and $\left(A_{1}, A_{2}\right)$ is hypercyclic. Lemma 2.1 implies that the sequence

$$
\left\{\left(\begin{array}{c}
\frac{n}{a}+\frac{m}{b} \\
a^{n} b^{m}
\end{array}\right): n, m \in \mathbb{N}\right\}
$$

is dense in $\mathbb{R}^{2}$. Observe that neither $|a|$ nor $|b|$ is equal to 1 . By taking the absolute value in the second coordinate and then applying the logarithmic function, we find that the sequence

$$
\left\{\left(\begin{array}{c}
\frac{n}{a}+\frac{m}{b} \\
n \ln |a|+m \ln |b|
\end{array}\right): n, m \in \mathbb{N}\right\}
$$

is dense in $\mathbb{R}^{2}$. Hence the sequence

$$
\left\{\left(\begin{array}{l}
n \frac{\ln |a|}{a}+m \frac{\ln |a|}{b} \\
n \frac{\ln |a|}{a}+m \frac{\ln |b|}{a}
\end{array}\right): n, m \in \mathbb{N}\right\}
$$

is dense in $\mathbb{R}^{2}$. Subtracting the second coordinate from the first one, we conclude that the sequence

$$
\left\{m\left(\frac{\ln |a|}{b}-\frac{\ln |b|}{a}\right): m \in \mathbb{N}\right\}
$$

is dense in $\mathbb{R}$, which is absurd. We proceed with the proof of assertion (ii). By Proposition 2.9 there exist $a \in \mathbb{R} \backslash \mathbb{Q}$ and $b \in \mathbb{C}$ such that the sequence $\left\{a^{n} b^{m}\right.$ : $n, m \in \mathbb{N}\}$ is dense in $\mathbb{C}$. Write $b=|b| e^{i \theta}$ and set

$$
A_{1}=\left(\begin{array}{cc}
a & 0 \\
0 & a
\end{array}\right), \quad A_{2}=\left(\begin{array}{cc}
|b| \cos \theta & -|b| \sin \theta \\
|b| \sin \theta & |b| \cos \theta
\end{array}\right) .
$$

Then we have

$$
A_{1}{ }^{n} A_{2}{ }^{m}=\left(\begin{array}{cc}
a^{n}|b|^{m} \cos m \theta & -a^{n}|b|^{m} \sin m \theta \\
a^{n}|b|^{m} \sin m \theta & a^{n}|b|^{m} \cos m \theta
\end{array}\right) .
$$

Applying in the above relation the vector $\left(\begin{array}{l}1 \\ 0\end{array}\right)$ and taking into account that the sequence $\left\{a^{n} b^{m}: n, m \in \mathbb{N}\right\}$ is dense in $\mathbb{C}$, we conclude that the sequence

$$
\left\{A_{1}{ }^{n} A_{2}{ }^{m}\left(\begin{array}{c}
1 \\
0
\end{array}\right): n, m \in \mathbb{N}\right\}=\left\{\left(\begin{array}{c}
a^{n}|b|^{m} \cos m \theta \\
a^{n}|b|^{m} \sin m \theta
\end{array}\right): n, m \in \mathbb{N}\right\}
$$

is dense in $\mathbb{R}^{2}$. Hence $\left(A_{1}, A_{2}\right)$ is hypercyclic. It is now easy to show that every non-zero vector in $\mathbb{R}^{2}$ is hypercyclic for $\left(A_{1}, A_{2}\right)$.

In order to prove the last assertion we follow a similar line of reasoning as above. That is, by Proposition 2.9 there exist $a, b \in \mathbb{C}$ such that the sequence $\left\{a^{n} b^{m}\right.$ : $n, m \in \mathbb{N}\}$ is dense in $\mathbb{C}$. Write $a=|a| e^{i \phi}, b=|b| e^{i \theta}$ and set

$$
A_{1}=\left(\begin{array}{cc}
|a| \cos \phi & -|a| \sin \phi \\
|a| \sin \phi & |a| \cos \phi
\end{array}\right), \quad A_{2}=\left(\begin{array}{cc}
|b| \cos \theta & -|b| \sin \theta \\
|b| \sin \theta & |b| \cos \theta
\end{array}\right) .
$$


A direct computation gives that $\left\{A_{1}{ }^{n} A_{2}{ }^{m}\left(\begin{array}{l}1 \\ 0\end{array}\right): n, m \in \mathbb{N}\right\}$ is equal to

$$
\left\{\left(\begin{array}{c}
|a|^{n}|b|^{m} \cos (n \phi+m \theta) \\
|a|^{n}|b|^{m} \sin (n \phi+m \theta)
\end{array}\right): n, m \in \mathbb{N}\right\}
$$

and by the choice of $a, b$ we conclude that the vector $\left(\begin{array}{l}1 \\ 0\end{array}\right)$ is hypercyclic for $\left(A_{1}, A_{2}\right)$. This completes the proof of the proposition.

Question 2.11. What is the minimum number of $2 \times 2$ matrices over $\mathbb{R}$ in Jordan form so that their tuple forms a hypercyclic operator?

2.2. The complex case. In what follows we will be writing $\Re(z)$ and $\Im(z)$ for the real and imaginary parts of a complex number $z$ respectively.

Proposition 2.12. There exist $a_{j} \in \mathbb{C}, j=1,2, \ldots, 8$ such that the sequence

$$
\left\{\left(\begin{array}{c}
\frac{k_{1}}{a_{1}}+\frac{k_{2}}{a_{2}}+\ldots+\frac{k_{8}}{a_{8}} \\
a_{1} k_{1} a_{2} k_{2} \ldots a_{8} k_{8}
\end{array}\right): k_{1}, k_{2}, \ldots, k_{8} \in \mathbb{N}\right\}
$$

is dense in $\mathbb{C}^{2}$.

Proof. The proof is in the same spirit as the proof of Proposition 2.7. Fix $a, b \in \mathbb{C}$ such that $-1<a<0, a+\frac{1}{a}, a-\frac{1}{a} \in \mathbb{R} \backslash \mathbb{Q}$ and $\left\{a^{n} b^{m}: n, m \in \mathbb{N}\right\}$ is dense in $\mathbb{C}$ (see Proposition 2.9). Let $z_{1}, z_{2} \in \mathbb{C}$ and $\epsilon>0$ be given. Then there exist $n, m \in \mathbb{N}$ such that $\left|a^{n} b^{m}-z_{2}\right|<\epsilon$. Note that

$$
a^{n} b^{m}=a^{n+k} b^{m} \frac{1}{a^{k}} 1^{s}(i a)^{\xi}\left(\frac{1}{i a}\right)^{\xi}(4 i)^{\rho}\left(-\frac{1}{4}\right)^{\rho}
$$

for every $k, s, \xi \in \mathbb{N}$ and $\rho \in 4 \mathbb{N}$. Note that $a+\frac{1}{a}<0$ and $a-\frac{1}{a}>0$. Hence, by Theorem 2.3, the sequence

$$
\left\{\xi\left(a-\frac{1}{a}\right)-\left(\frac{\rho}{4}\right): \xi \in \mathbb{N}, \rho \in 4 \mathbb{N}\right\}
$$

is dense in $\mathbb{R}$. As a result, there exist $\xi \in \mathbb{N}$ and $\rho \in 4 \mathbb{N}$ such that

$$
\left|\Im\left(i \xi\left(a-\frac{1}{a}\right)-i\left(\frac{\rho}{4}\right)\right)-\Im\left(z_{1}-\frac{n}{a}-\frac{m}{b}\right)\right|<\epsilon ;
$$

i.e. we have that

$$
\left|\Im\left(\frac{n}{a}+\frac{m}{b}+i \xi\left(a-\frac{1}{a}\right)-i\left(\frac{\rho}{4}\right)\right)-\Im\left(z_{1}\right)\right|<\epsilon .
$$

By Remark 2.4, the sequence

$$
\left\{k\left(a+\frac{1}{a}\right)+s: k, s \in \mathbb{N}\right\}
$$

is dense in $\mathbb{R}$. Hence, there exist $k, s \in \mathbb{N}$ such that

$$
\left|k\left(a+\frac{1}{a}\right)+s-\left(4 \rho+\Re\left(z_{1}-\frac{n}{a}-\frac{m}{b}\right)\right)\right|<\epsilon ;
$$

i.e. we have that

$$
\left|\Re\left(\frac{n}{a}+\frac{m}{b}+k\left(a+\frac{1}{a}\right)-4 \rho+s\right)-\Re\left(z_{1}\right)\right|<\epsilon .
$$


But this means that the real and imaginary parts of the complex number

$$
\frac{n}{a}+\frac{m}{b}+k\left(a+\frac{1}{a}\right)+s+i \xi\left(a-\frac{1}{a}\right)-i \frac{\rho}{4}-4 \rho
$$

are within $\epsilon$ of the real and imaginary parts of $z_{1}$. Hence, setting $a_{1}=a, a_{2}=$ $b, a_{3}=\frac{1}{a}, a_{4}=1, a_{5}=i a, a_{6}=\frac{1}{i a}, a_{7}=4 i, a_{8}=-\frac{1}{4}$, we prove the result.

Proof of Theorem 1.3. By Proposition 2.12, Lemma 2.1 and Remark 2.2 the assertion follows.

Example 2.13. Fix $a, b \in \mathbb{C}$ such that $-1<a<0, a+\frac{1}{a}, a-\frac{1}{a} \in \mathbb{R} \backslash \mathbb{Q}$ and $\left\{a^{n} b^{m}: n, m \in \mathbb{N}\right\}$ is dense in $\mathbb{C}$. From the above it is evident that the 8 -tuple of $2 \times 2$ matrices in Jordan form over $\mathbb{C}$ given by

$$
\begin{aligned}
& \left(\begin{array}{cc}
a & 1 \\
0 & a
\end{array}\right),\left(\begin{array}{cc}
b & 1 \\
0 & b
\end{array}\right),\left(\begin{array}{cc}
\frac{1}{a} & 1 \\
0 & \frac{1}{a}
\end{array}\right),\left(\begin{array}{ll}
1 & 1 \\
0 & 1
\end{array}\right), \\
& \left(\begin{array}{cc}
i a & 1 \\
0 & i a
\end{array}\right),\left(\begin{array}{cc}
\frac{1}{i a} & 1 \\
0 & \frac{1}{i a}
\end{array}\right),\left(\begin{array}{cc}
4 i & 1 \\
0 & 4 i
\end{array}\right),\left(\begin{array}{cc}
-\frac{1}{4} & 1 \\
0 & -\frac{1}{4}
\end{array}\right)
\end{aligned}
$$

is hypercyclic.

Question 2.14. What is the minimum number of $2 \times 2$ matrices over $\mathbb{C}$ in Jordan form so that their tuple forms a hypercyclic operator?

\section{Products of $3 \times 3$ Matrices}

In this section we start with the following special case of Corollary 3.5 in 4, due to Feldman, which will be of use to us in the following.

Proposition 3.1 (Feldman). If $b_{1}, b_{2} \in \mathbb{D} \backslash\{0\}$, then there exists a dense set $\Delta \subset \mathbb{C} \backslash \mathbb{D}$ such that for every $a_{1}, a_{2} \in \Delta$ the sequence

$$
\left\{\left(\begin{array}{c}
a_{1}{ }^{n} b_{1}{ }^{m} \\
a_{2}{ }^{n} b_{2}{ }^{l}
\end{array}\right): n, m, l \in \mathbb{N}\right\}
$$

is dense in $\mathbb{C}^{2}$.

In order to handle products of $3 \times 3$ matrices, we establish the following:

Corollary 3.2. There exist $a \in \mathbb{C}$ and $b, c, d \in \mathbb{R}$ such that the sequence

$$
\left\{\left(\begin{array}{c}
a^{n} b^{m} \\
c^{n} d^{l}
\end{array}\right): n, m, l \in \mathbb{N}\right\}
$$

is dense in $\mathbb{C} \times \mathbb{R}$.

Proof. Fix two real numbers $b_{1}, b_{2}$ with $b_{1}, b_{2} \in(0,1)$. By Proposition 3.1 there exist $a_{1}, a_{2} \in \mathbb{C} \backslash \mathbb{D}$ such that the sequence

$$
\left\{\left(\begin{array}{c}
a_{1}{ }^{n} b_{1}{ }^{m} \\
a_{2}{ }^{n} b_{2}{ }^{l}
\end{array}\right): n, m, l \in \mathbb{N}\right\}
$$

is dense in $\mathbb{C}^{2}$. Define $a=a_{1}, b=b_{1}, c=\left|a_{2}\right|$ and $d=-\sqrt{b_{2}}$. Observe that the sequence

$$
\left\{\left(\begin{array}{c}
a^{n} b^{m} \\
c^{n} b_{2}^{l}
\end{array}\right): n, m, l \in \mathbb{N}\right\}
$$

is dense in $\mathbb{C} \times[0,+\infty)$. Take $z \in \mathbb{C}$ and $x \in \mathbb{R}$. 
Case I. $x \geq 0$.

Then there exist sequences of positive integers $\left\{n_{k}\right\},\left\{m_{k}\right\},\left\{l_{k}\right\}$ such that

$$
a^{n_{k}} b^{m_{k}} \rightarrow z \text { and } c^{n_{k}} b_{2}{ }^{l_{k}} \rightarrow x .
$$

Since $b_{2}{ }^{l_{k}}=d^{2 l_{k}}$ we get $c^{n_{k}} d^{2 l_{k}} \rightarrow x$.

Case II. $x<0$.

Then there exist sequences of positive integers $\left\{n_{k}\right\},\left\{m_{k}\right\},\left\{l_{k}\right\}$ such that

$$
a^{n_{k}} b^{m_{k}} \rightarrow z \text { and } c^{n_{k}} b_{2}^{l_{k}} \rightarrow \frac{x}{d} .
$$

The last implies that $c^{n_{k}} d^{2 l_{k}+1} \rightarrow x$. This completes the proof of the corollary.

The main result of this section is to prove Theorem 1.1 for $n=3$. This is stated and proved below.

Proposition 3.3. There exist 3 tuples $\left(A_{1}, A_{2}, A_{3}\right)$ of $3 \times 3$ matrices over $\mathbb{R}$ such that $\left(A_{1}, A_{2}, A_{3}\right)$ is hypercyclic.

Proof. By Corollary 3.2 there exist $a \in \mathbb{C}$ and $b, c, d \in \mathbb{R}$ such that the sequence

$$
\left\{\left(\begin{array}{c}
a^{n} b^{m} \\
c^{n} d^{l}
\end{array}\right): n, m, l \in \mathbb{N}\right\}
$$

is dense in $\mathbb{C} \times \mathbb{R}$. Write $a=|a| e^{i \theta}$ and set

$$
\begin{gathered}
A_{1}=\left(\begin{array}{ccc}
|a| \cos \theta & -|a| \sin \theta & 0 \\
|a| \sin \theta & |a| \cos \theta & 0 \\
0 & 0 & c
\end{array}\right), A_{2}=\left(\begin{array}{ccc}
b & 0 & 0 \\
0 & b & 0 \\
0 & 0 & 1
\end{array}\right) \text { and } \\
A_{3}=\left(\begin{array}{lll}
1 & 0 & 0 \\
0 & 1 & 0 \\
0 & 0 & d
\end{array}\right) .
\end{gathered}
$$

Then we have

$$
A_{1}{ }^{n} A_{2}{ }^{m} A_{3}{ }^{l}=\left(\begin{array}{ccc}
|a|^{n} b^{m} \cos n \theta & -|a|^{n} b^{m} \sin n \theta & 0 \\
|a|^{n} b^{m} \sin n \theta & |a|^{n} b^{m} \cos n \theta & 0 \\
0 & 0 & c^{n} d^{l}
\end{array}\right),
$$

which in turn gives

$$
A_{1}{ }^{n} A_{2}{ }^{m} A_{3}{ }^{l}\left(\begin{array}{c}
1 \\
0 \\
1
\end{array}\right)=\left(\begin{array}{c}
|a|^{n} b^{m} \cos n \theta \\
|a|^{n} b^{m} \sin n \theta \\
c^{n} d^{l}
\end{array}\right) .
$$

The last and the choice of $a, b, c, d$ imply that $\left(A_{1}, A_{2}, A_{3}\right)$ is hypercyclic with $\left(\begin{array}{l}1 \\ 0 \\ 1\end{array}\right)$ being a hypercyclic vector for $\left(A_{1}, A_{2}, A_{3}\right)$. 


\section{Proof of Theorem 1.1}

By Proposition 2.10, there exist $2 \times 2$ matrices $B_{1}$ and $B_{2}$ such that $\left(B_{1}, B_{2}\right)$ is hypercyclic.

Case I. $n=2 k$ for some positive integer $k$. For $k=1$ the result follows by Proposition 2.10. Assume that $k>1$. Each $A_{j}$ will be constructed by blocks of $2 \times 2$ matrices. Let $I_{2}$ be the $2 \times 2$ identity matrix. We will be using the notation $\operatorname{diag}\left(D_{1}, D_{2}, \ldots, D_{n}\right)$ to denote the diagonal matrix with diagonal entries the block matrices $D_{1}, D_{2}, \ldots, D_{n}$. Define $A_{1}=\operatorname{diag}\left(B_{1}, I_{2}, \ldots, I_{2}\right), A_{2}=$ $\operatorname{diag}\left(B_{2}, I_{2}, \ldots, I_{2}\right), A_{3}=\operatorname{diag}\left(I_{2}, B_{1}, I_{2}, \ldots, I_{2}\right), A_{4}=\operatorname{diag}\left(I_{2}, B_{2}, I_{2}, \ldots, I_{2}\right)$ and so on up to $A_{n-1}=\operatorname{diag}\left(I_{2}, \ldots, I_{2}, B_{1}\right), A_{n}=\operatorname{diag}\left(I_{2}, \ldots, I_{2}, B_{2}\right)$.

It is now easy to check that $\left(A_{1}, A_{2}, \ldots, A_{n}\right)$ is hypercyclic and furthermore that the set $H C\left(\left(A_{1}, A_{2}, \ldots, A_{n}\right)\right)$ is

$$
\left\{\left(x_{1}, x_{2}, \ldots, x_{n}\right) \in \mathbb{R}^{n}: x_{2 j-1}^{2}+x_{2 j}^{2} \neq 0, \forall j=1,2, \ldots, k\right\} .
$$

Case II. $n=2 k+1$ for some positive integer $k$. If $k=1$ the result follows by Proposition 3.3. Suppose $k>1$. For simplicity we treat the case $k=2$, since the general case follows by similar arguments. By Proposition 3.3 there exist $C_{1}, C_{2}, C_{3}, 3 \times 3$ matrices such that $\left(C_{1}, C_{2}, C_{3}\right)$ is hypercyclic. Let $I_{3}$ be the $3 \times 3$ identity matrix. Define $A_{1}=\operatorname{diag}\left(B_{1}, I_{3}\right), A_{2}=\operatorname{diag}\left(B_{2}, I_{3}\right), A_{3}=\operatorname{diag}\left(I_{2}, C_{1}\right)$, $A_{4}=\operatorname{diag}\left(I_{2}, C_{2}\right)$ and $A_{5}=\operatorname{diag}\left(I_{2}, C_{3}\right)$.

It can easily be shown that $\left(A_{1}, A_{2}, \ldots, A_{5}\right)$ is hypercyclic. The details are left to the reader.

\section{ACKNOWLEDGEMENT}

The authors would like to thank the anonymous referee for helpful and constructive comments.

\section{REFERENCES}

[1] F. Bayart, É. Matheron, Topics in linear dynamics, Cambridge Tracts in Math. (to appear).

[2] N. S. Feldman, Hypercyclic Tuples of Operators. Mini-workshop: Hypercyclicity and Linear Chaos. Abstracts from the mini-workshop held August 13-19, 2006. Organized by Teresa Bermúdez, Gilles Godefroy, Karl-G. Grosse-Erdmann and Alfredo Peris. Oberwolfach Reports, Vol. 3, no. 3. Oberwolfach Rep. 3 (2006), no. 3, 2227-2276. MR2332177

[3] N. S. Feldman, Hypercyclic pairs of coanalytic Toeplitz operators, Integral Equations Operator Theory 58 (2007), 153-173. MR.2324885 (2008d:47057)

[4] N. S. Feldman, Hypercyclic tuples of operators and somewhere dense orbits, J. Math. Anal. Appl. 346 (2008), 82-98.

[5] K.-G. Grosse-Erdmann, Universal families and hypercyclic operators, Bull. Amer. Math. Soc. 36 (1999), 345-381. MR 1685272 (2000c:47001)

[6] K.-G. Grosse-Erdmann, Recent developments in hypercyclicity, RACSAM Rev. R. Acad. Cienc. Exactas Fis. Nat. Ser. A Mat. 97 (2003), 273-286. MR2068180 (2005c:47010)

[7] K.-G. Grosse-Erdmann, Dynamics of linear operators, Topics in complex analysis and operator theory, 41-84, Univ. Malaga, Malaga, 2007. MR2394656 
[8] G. H. Hardy and E. M. Wright, An Introduction to the Theory of Numbers, 5th edition, The Clarendon Press, Oxford University Press, New York, 1979. MR0568909 (81i:10002)

[9] L. Kérchy, Cyclic properties and stability of commuting power bounded operators, Acta Sci. Math. (Szeged) 71 (2005), 299-312. MR2160368(2006e:47014)

Department of Mathematics, University of Crete, Knossos Avenue, GR-714 09 Heraklion, Crete, Greece

E-mail address: costakis@math.uoc.gr

Department of Computer Science and Engineering, European University Cyprus, 6 Diogenes Street, Engomi, P.O. Box 22006, 1516 Nicosia, Cyprus

E-mail address: d.hadjiloucas@euc.ac.cy

Fakultät für Mathematik, SFB 701, Universität Bielefeld, Postfach 100131, D-33501 Bielefeld, Germany

E-mail address: amanouss@math.uni-bielefeld.de 